\title{
An efficient CSMA-CA algorithm for IEEE 802.15.4 wireless sensor networks
}

\begin{abstract}
Different Medium Access Control (MAC) Protocols for Wireless Sensor Networks (WSNs) have been proposed over the last few years. IEEE 802.15.4 MAC protocol standard is designed for low-power, low-cost, and low-rate Wireless Personal Area Networks (LRWPANs). The contention period of IEEE 802.15.4 standard consists of Contention Access Period (CAP) and Contention Free Period (CFP). CAP utilizes Carrier Sense Multiple Access with Collision Avoidance (CSMA-CA) algorithm for its contention mechanism and channel access. In such networks, the range of backoff exponent is limited to very small values. Problem arises with increased probability of devices choosing the same number of backoff period (BPs). In return, this problem affects the channel utilization and packet collisions. In this paper, we proposed an efficient and adaptive backoff algorithm (EBA) to minimize the collisions among the contending nodes. While CSMA-CA delays for a limited number of backoff ranges, EBA is designed to enhance the performance of CSMA-CA by choosing the appropriate window size based on the collisions determined by the node. Furthermore, to increase the efficiency of EBA we split the backoff period into sub-backoff period called temporary backoff (TB) and next temporary backoff (NTB). During the implementation of EBA, the node choose the TB and NTB value randomly which is represent $10 \%$ to $50 \%$ of the actual backoff delay value instead of choosing backoff exponent (BE) randomly as in the standard. In this case, we decrease the probability of choosing the same number of backoff exponent randomly selected by nodes and minimize the probability of collision. Hence, we give better opportunities for successful packet transmissions. Simulation results demonstrated and compared the performance of EBA with that of IEEE 802.15.4 standard. According to the results obtained, EBA achieved better performance than the 802.15.4 standard in terms of throughput, delivery ration, and power consumption.
\end{abstract}

Keyword: IEEE 802.15.4; CSMA-CA; MAC; Backoff algorithm; WSNs 